\title{
Propuesta de mejora de procesos para la cadena de suministro de una imprenta
}

\section{Process improvement proposal for the supply chain of a printing press}

\author{
(iD) 'Edwin Wilfredo Sencara Sencara (iD ' Gustavo Adolfo Montoya Cárdenas (iD ²José Luis Carrión Nin, \\ iD 3Jorge Rafael Diaz Dumont.
}

Universidad Nacional Mayor de San Marcos. Lima, Perú.

Universidad César Vallejo. Lima, Perú. ${ }^{2}$

Universidad Nacional Autónoma de Tayacaja "Daniel Hernández Morillo". Pampas, Perú. ${ }^{3}$

\section{Resumen}

El presente artículo tuvo como problemática abordar el tema del incumplimiento con el plazo de entrega de los pedidos, que ocasiona insatisfacción de los clientes de la empresa. Por ello, se aplicó como herramienta de mejora, la metodología de los 7 pasos. Esta metodología logra identificar el problema principal que es la demora en la entrega inoportuna de pedidos y las causas raíces, se identificaron mediante herramientas de calidad como Pareto, Ishikawa y Árbol causal. Asimismo, se determinó el comportamiento del proceso empleando el Supplier Inputs Process Outputs Customers (SIPOC), fichas de control e indicadores. Por otro lado, se evaluó las mejores propuestas de solución para implementarlas, donde se plantea el ordenamiento, procedimientos y los equipos a implementar, que garanticen la reducción de tiempo en el proceso de la cadena de suministro y el respectivo control por medio de indicadores, logrando la reducción de tiempo de 33\%, es decir, de 9 días a 6 días. Por último, se presentan las conclusiones resultantes del estudio de mejora.

Palabras clave: 5S, lean Manufacturing, picking.

\section{Abstract}

This article was problematic to address the issue of non-compliance with the delivery of orders, which causes dissatisfaction of the company's customers. Therefore, the 7-step methodology was applied as an improvement tool. This methodology manages to identify the main problem that is the delay in the untimely delivery of orders and the root causes, were identified by quality tools such as Pareto, Ishikawa and Causal tree. Likewise, the behavior of the process was determined using the SIPOC, control sheets and indicators. On the other hand, the best

Recibido:

Marzo 2020

Aceptado: Junio 2020 solution proposals to implement them were evaluated, where the ordering, procedures and equipment to be implemented are proposed, which guarantee the reduction of time in the supply chain process and the respective control through indicators, achieving the 33\% time reduction, that is, from 9 days to 6 days. Fina$\| y$, the conclusions resulting from the improvement study are presented.

Keywords: 5S, lean Manufacturing, picking. 


\section{INTRODUCCIÓN}

En la actualidad, muchas de las empresas tienen como objetivo una estabilidad económica. Por ello, existe la competitividad para lograr mantenerse en el mercado y la industria gráfica no es la excepción, ya que en el mercado ha evolucionado de manera exponencial. Por eso, están obligando a las empresas a trabajar con mayor rapidez, para afrontar los cambios demandados por el mercado y eficiencia en sus operaciones. Esto permitirá a las empresas satisfacer las necesidades de sus clientes, cumpliendo sus requisitos de calidad, cantidad y tiempos de respuesta. En ese sentido, las empresas que no se acoplan a las exigencias del mercado, irán camino al fracaso.

Por otro lado, el presente trabajo de investigación, está relacionado con el tiempo de entrega de pedidos, de la empresa de imprenta AYEMI E.I.R.L; ya que se recibe frecuentemente las quejas sobre la insatisfacción de los clientes, por sus pedidos que no llegan de acuerdo a lo programado. La empresa ha recibido 537 pedidos del año 2018, entregando sus pedidos fuera del tiempo establecido.

Asimismo, el problema de estudio es el incumpliendo con el plazo de entrega de los pedidos. Esto genera malestar en los clientes y merma el prestigio de la empresa. Además, si la empresa sigue manteniendo las entregas tardías incide a que baje las ventas y se pierdan los clientes, por esta razón, es de interés plantear alternativas de solución al problema expuesto. En este estudio, se implementó la metodología de los 7 pasos (mejora continua), que desarrolló mejoras en los diferentes procesos de la organización, asimismo, para aumentar la satisfacción del cliente mediante el cumplimiento de sus requisitos.

Ramírez y Peña (2011). En el siguiente diagrama se esquematiza el flujo de órdenes de producción.

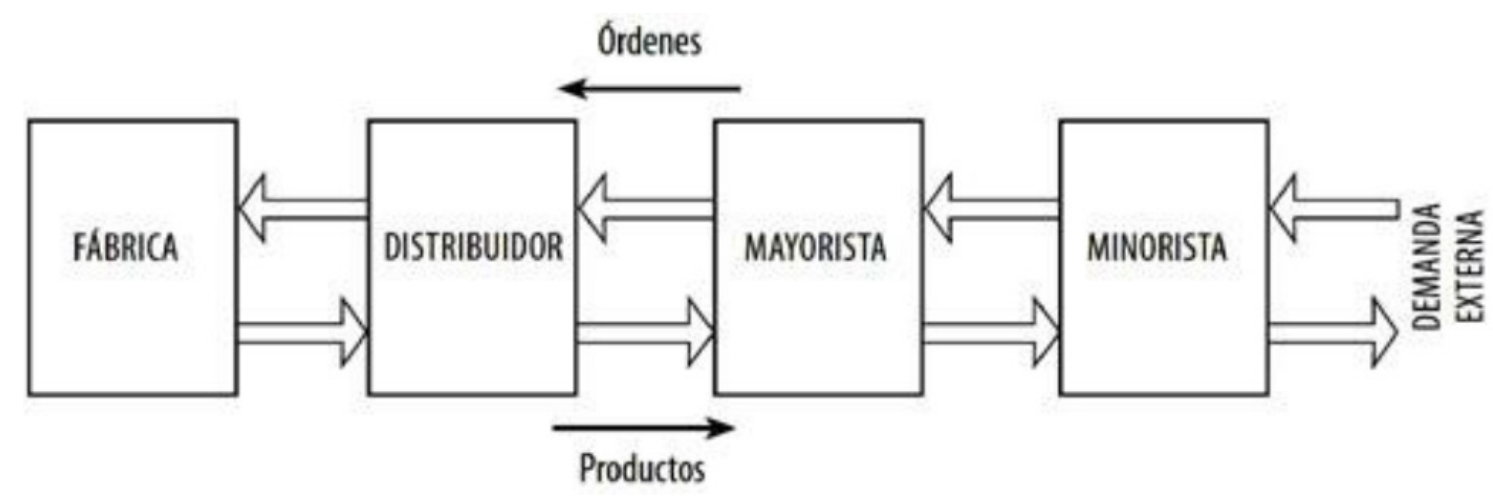

Figura 1. Flujo de órdenes y productos en el modelo.

Fuente: Ramírez y Peña (2011)

Camacho, Gómez y Monroy (2012), plantean la importancia de la Cadena de Suministros: "Cuando se habla del suministro se hace referencia a la materia prima, respondiendo a las preguntas cómo, dónde y cuándo se conseguirán y se suministrarán al proceso. En la fabricación se transforma la materia prima en el producto terminado, luego viene la distribu- ción que es donde se logra entregar al cliente el producto final, lo cual se puede hacer por medio de mercados mayoristas y minoristas, entre otros" (p. s/n).

Para Vianchá (2014) "Planear la Cadena de Suministros Alimentaria requiere del diseño de modelos de gestión que logren identificar 
ampliamente la configuración del territorio, teniendo en cuenta aspectos como el tamaño [...], las condiciones de producción primaria, la tecnología, la demanda del mercado, la capacidad financiera y las prácticas de gestión, entre otros" (p. s/n).

Para Cano, Orué, Martínez, Mayett, y López. (2015) "Es un hecho el constante cambio en los gustos y hábitos de los consumidores en la demanda de productos. Esta situación trae como consecuencia que los consumidores y los productos consumidos no estén en equilibrio, y se modifique el interés de satisfacer las nuevas expectativas del mercado" (p. s/n).

Según Salas, Maiguel y Acevedo (2017). Existen 5 pasos para la gestión de inventarios y su integración con la cadena de suministros, estos son: "Paso 1: Definición de políticas para la integración y colaboración, Paso 2: Planificación colaborativa, Paso 3: Integración de procesos claves y críticos, Paso 3: Integración de procesos claves y críticos, Paso 4: Medición del desempeño, Paso 5: Elaboración de planes de acción" (p. s/n).

Según Salas, Obredor, Mercado (2019) "El abastecimiento se consolida con la materia prima e insumos para la transformación en productos terminados. Por esto se garantiza la calidad de los insumos, y la participación de los proveedores para contribuir con la productividad en los procesos organizaciones y la competitividad" (p. s/n); en este orden de ideas los procesos logísticos se entienden como parte de un sistema en donde la retroalimentación juega un papel importante en la mejora continua, para ello es importante considerar:

\section{Descripción de la empresa}

La imprenta AYEMI E.I.R.L. se destacó en la industria gráfica por su arduo trabajo y gran capital humano, fue fundada el 26 de octubre de 2007.

Actualmente, la empresa trabaja con más de 15 trabajadores, que brindan una atención cordial a sus clientes a nivel nacional. Además, la imprenta AYEMI E.I.R.L se especializa en impresiones, que se plasman en diversos tipos de productos tales como: cuadernos, libretas, impresión de libros, papel milimetrado, fichas, cuadernos de dibujos, entre otros.

\section{Determinación de causas de problemas}

En el diagrama de Pareto que se aprecia en la figura 1, el problema que presenta la empresa de estudio es el incumpliendo con el plazo de entrega de los pedidos, de acuerdo a los reclamos realizados por los clientes.

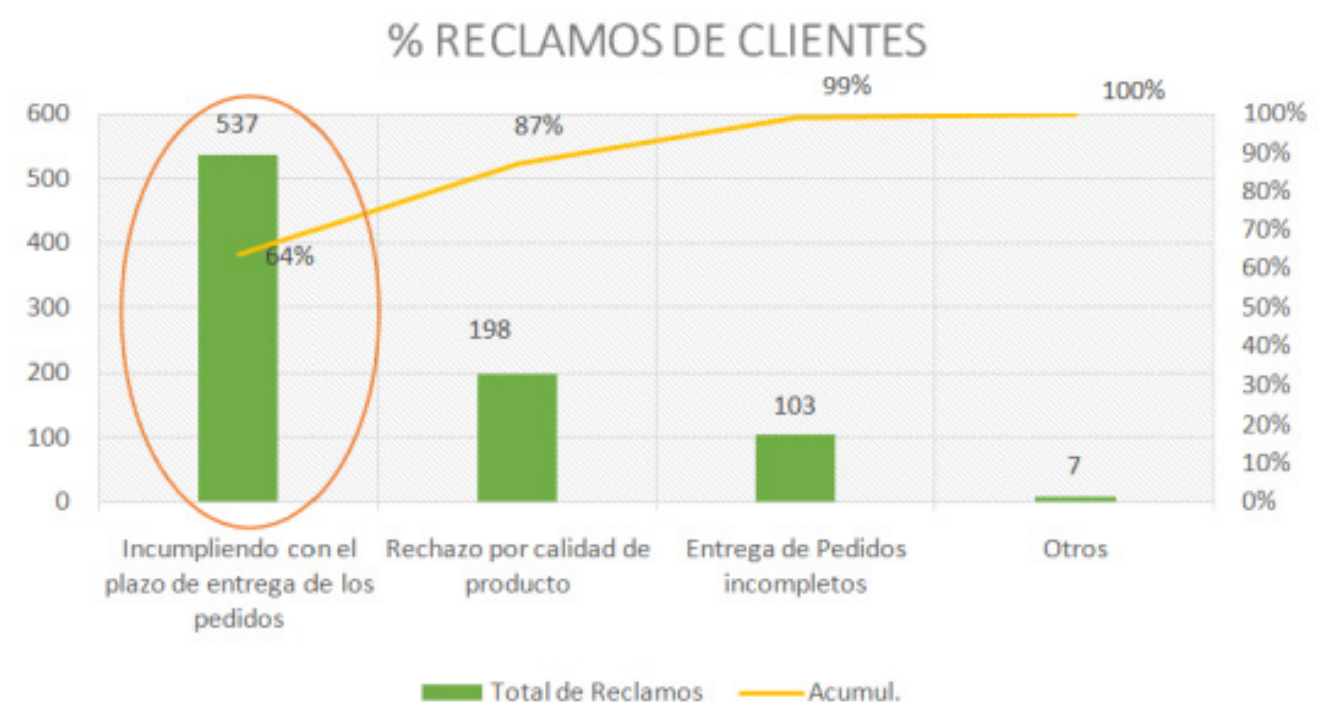

Figura 2. Diagrama Pareto - reclamo de clientes 
El objetivo de este estudio, es cumplir con todas las órdenes de trabajo entregadas por nuestro cliente en la fecha establecida, logrando un incremento de la facturación y la productividad en la empresa.
Con respecto al problema principal, generó un impacto económico en el año 2018 por un monto total de S/. 104,864 en el que son generados por pedidos no atendidos a tiempo. En la Figura 2 , se puede apreciar la evolución del incumplimiento de los pedidos de los años anteriores.

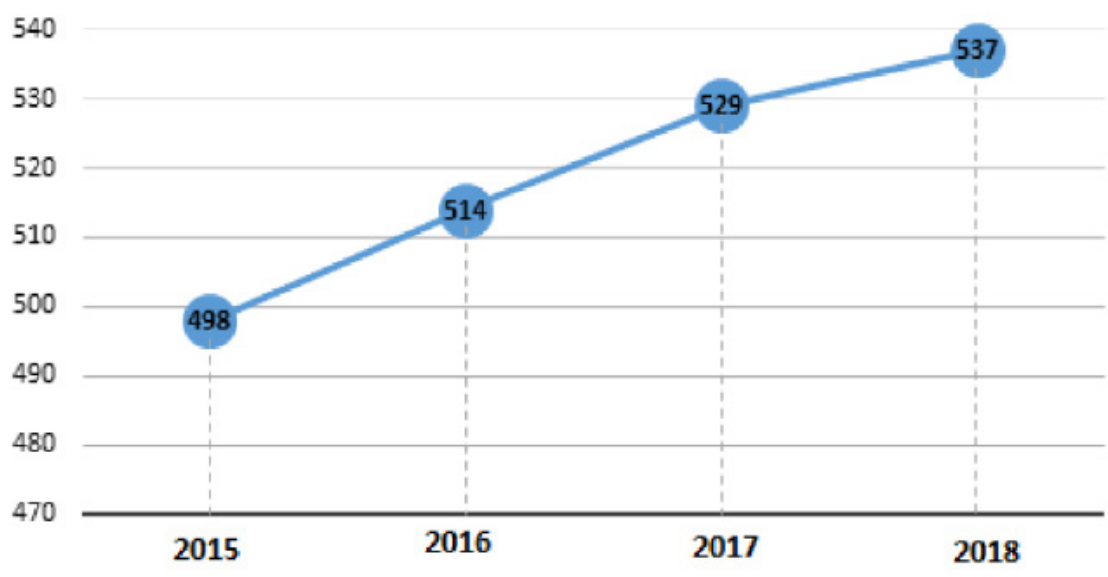

Figura 3. Evolución de los Pedidos incumplidos en los años 2015, 2016, 2017 y 2018.

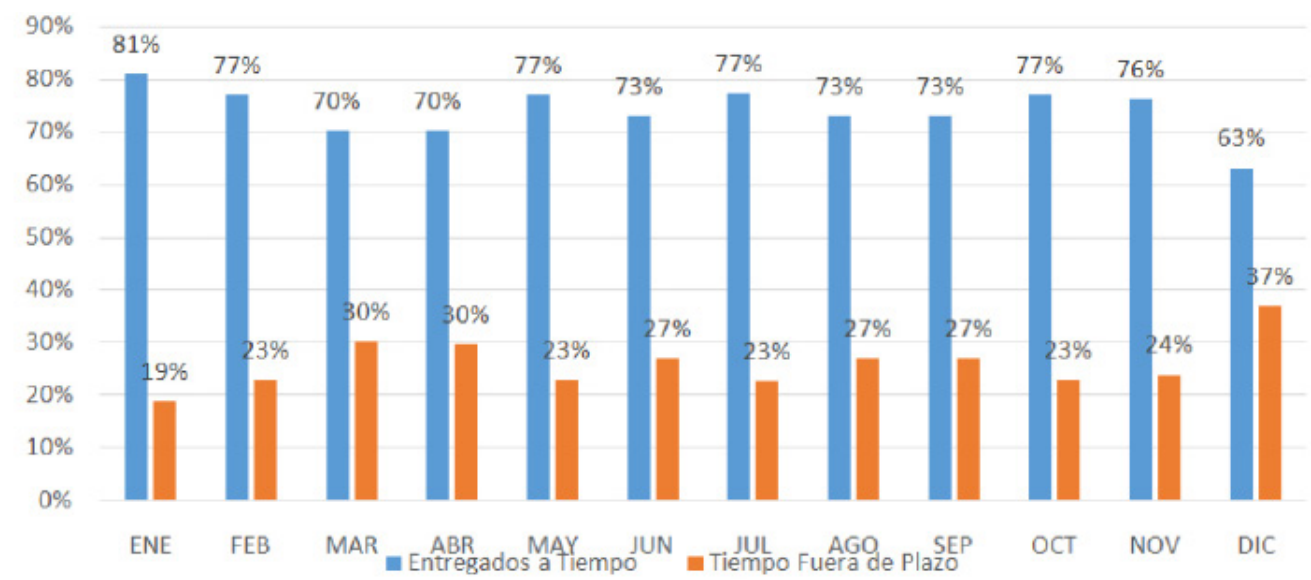

Figura 4. \% Pedidos Entregados a Tiempo vs \% Pedidos Entregados fuera de plazo mensual del año.

\section{Propuestas de mejora de procesos}

\section{Paso $\mathbf{N}^{\circ}$ 1: Seleccionar el problema}

En este paso se determina el problema de estudio, que es el incumpliendo con el plazo de entrega de los pedidos, lo que ocasiona la insatisfacción de los clientes y la empresa se ve 
afectada en los ingresos económicos, prestigio e imagen institucional, disminución de ventas, pérdida de clientes y los incrementos de costos operativos, por realizar horas extras, errores, etc. En la tabla 1, se selecciona el problema del incumplimiento con el plazo de entrega de los pedidos, que tiene mayor frecuencia de demora 64\%; sin embargo, existen otros problemas que se evidencian y que será necesarios resolverlos una vez atendidos los más preponderantes, puesto que generan pérdidas que se irán acumulando conforme transcurra el tiempo.

Tabla 1. Frecuencia de procesos que demora

\begin{tabular}{|l|c|c|c|}
\hline \multicolumn{1}{|c|}{$\begin{array}{c}\text { Los Motivos de Reclamo de los } \\
\text { Clientes }\end{array}$} & $\begin{array}{c}\text { Total de } \\
\text { Reclamos }\end{array}$ & $\begin{array}{c}\% \text { de } \\
\text { Reclamos }\end{array}$ & Acumulado \\
\hline $\begin{array}{l}\text { Incumpliendo con el plazo de } \\
\text { entrega de los pedidos }\end{array}$ & 537 & $64 \%$ & $64 \%$ \\
\hline Rechazo por calidad de producto & 198 & $23 \%$ & $87 \%$ \\
\hline Entrega de Pedidlos incompletos & 103 & $12 \%$ & $99 \%$ \\
\hline Otros & 7 & $1 \%$ & $100 \%$ \\
\hline \multicolumn{1}{|c|}{ Total } & $\mathbf{8 4 5}$ & $100 \%$ & \\
\hline
\end{tabular}

Fuente: La empresa.

Paso $N^{\circ}$ 2: Comprender la situación y establecer metas

El problema principal es el incumplimiento con el plazo de entrega de los pedidos. En ese sentido, en la Tabla 1, se muestra año por año sobre las cantidades de pedidos incumplidos.

Tabla 2. Número de pedidos incumplidos en los años de estudio

\begin{tabular}{lcccc}
\hline \multicolumn{1}{c}{ Mes } & $\mathbf{2 0 1 5}$ & $\mathbf{2 0 1 6}$ & $\mathbf{2 0 1 7}$ & $\mathbf{2 0 1 8}$ \\
\hline Enero & 32 & 35 & 37 & 37 \\
Febrero & 30 & 43 & 47 & 45 \\
Marzo & 29 & 36 & 44 & 33 \\
Abril & 37 & 39 & 41 & 44 \\
Mayo & 36 & 38 & 52 & 41 \\
Junio & 44 & 42 & 40 & 42 \\
Julio & 43 & 42 & 36 & 35 \\
Agosto & 44 & 39 & 34 & 44 \\
Septiembre & 41 & 38 & 44 & 41 \\
Octubre & 51 & 45 & 41 & 52 \\
Noviembre & 57 & 58 & $\mathbf{5 8}$ & 60 \\
Diciembre & 54 & 59 & 54 & 62 \\
\multicolumn{1}{c}{ Total } & $\mathbf{4 9 8}$ & $\mathbf{5 1 4}$ & $\mathbf{5 2 9}$ & $\mathbf{5 3 7}$ \\
\hline
\end{tabular}


Con respecto, al impacto económico que es generado por el incumplimiento con el plazo de entrega de los pedidos, se puede observar en la Figura 3.

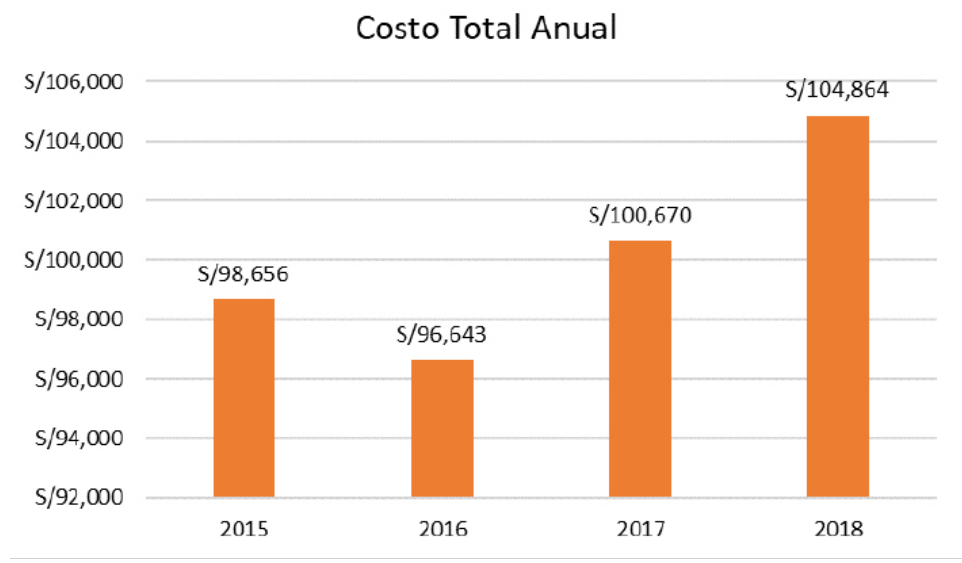

Figura 5. Costo anual por incumplimiento con el plazo de entrega de los pedidos

\section{Paso 3: Analizar Causas del problema}

Con respecto al problema principal, sobre el kawa, identificando las causas que ocasionan incumplimiento con el plazo de entrega de al problema principal, que se puede aprecia los pedidos, se elaboró el diagrama de Ishien la Figura 4.

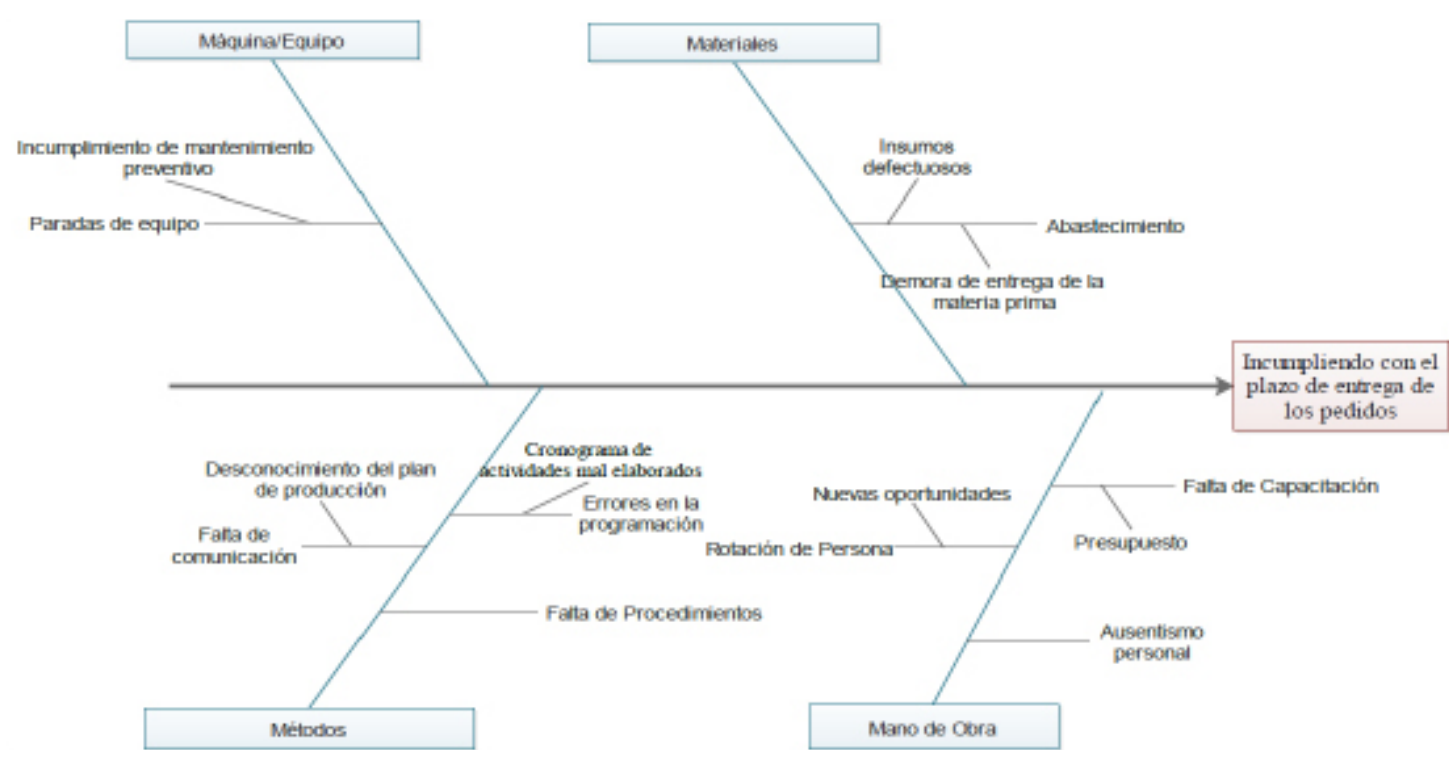

Figura 6. Diagrama de causa - efecto del incumplimiento con el plazo de entrega de los pedidos

Asimismo, se desarrolló el Diagrama de Pareto para la identificación de las causas a solucionar, como se observa en la figura 5. Donde se concluye, en la identificación las siguientes causas principales:

- Demora de abastecimiento de insumos.

- Paradas de Máquinas

- Demora en el proceso de empaquetado 


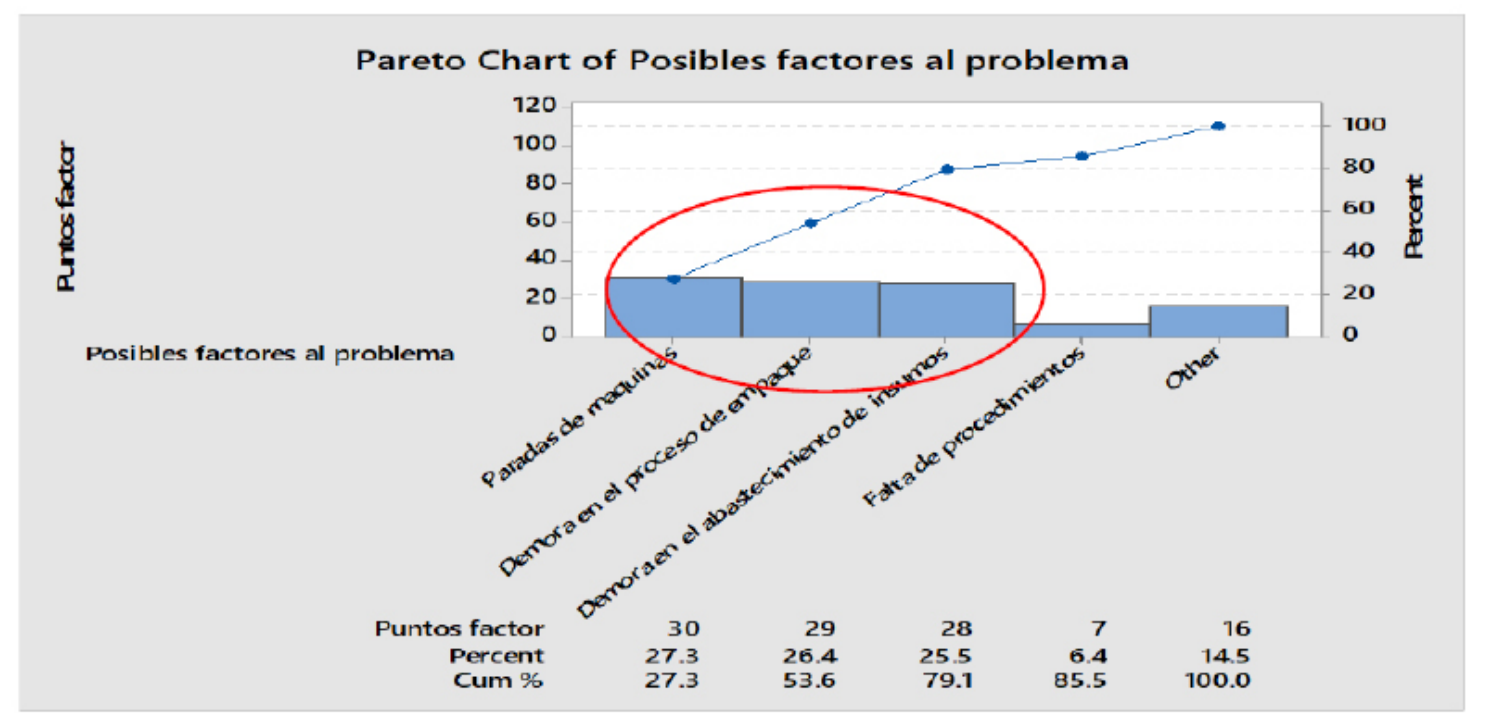

Figura 7. Diagrama de Pareto

Paso $N^{\circ}$ 4: Proponer, seleccionar y programar soluciones

Para la Imprenta AYEMI E.I.R.L. se estableció las alternativas de solución a la causa raíz, que afectan al problema principal de la entrega inoportuna, que son las siguientes:

- Disminuir el periodo de recepción de insumos.

- Disminuir el tiempo de parada de las maquinas (puede haber tiempo planificado y tiempo de paradas extraordinarias, innecesarias o indeseadas).

- Disminuir el tiempo de empaque

Para dar solución a las causas principales se propone la implementación de las 5S, que es método de gestión japonés: se fundamenta en cinco principios simples: Seiri, (clasificación), Seiton (orden), Seiso (limpieza), Seikesu (estandarizar), Shitsuke (mantener la disciplina).

- Implementación de las 5S.

Tabla 3. Cronograma de implementación del proyecto

\begin{tabular}{|c|c|c|c|c|c|c|c|c|c|c|c|c|}
\hline \multirow[b]{2}{*}{ Item } & & & \multicolumn{10}{|c|}{ Año 2019} \\
\hline & & ACTIVIDAD & Enero & Febrero & Marzo & Abril & Mayo & Junio & Julio & Agosto & Setiembre & Octubre \\
\hline $\mathrm{A}$ & Preliminar & Etapa preliminar & & & & & & & & & & \\
\hline 1 & Planear & $\begin{array}{l}\text { Etapa de desarrollo de la } \\
\text { metodología }\end{array}$ & & & & & & & & & & \\
\hline 2 & Ejecutar & $\begin{array}{l}\text { Implementación las } \\
\text { mejoras }\end{array}$ & & & & & & & & & & \\
\hline 3 & Verificar & $\begin{array}{l}\text { Documentar los procesos } \\
\text { mejoras }\end{array}$ & & & & & & & & & & \\
\hline 4 & Actuar & Compartir buenas practicas & & & & & & & & & & \\
\hline
\end{tabular}




\section{Paso $\mathrm{N}^{\circ}$ 5: Implementar y verificar resultados}

\section{Etapa 1: Implementación de la Clasificación-SEIRI}

Se procede a identificar, registrar y hacer un inventario de las cosas útiles del área, posteriormente se etiquetará de color rojo los artículos que no se requieran en el área y se procede según la indicación si es para destrucción, remate, etc. Ver Tabla 4.

Tabla 4. Modelo de Tarjeta Roja

\begin{tabular}{|c|c|c|}
\hline \multicolumn{3}{|c|}{ TARJETA ROJA } \\
\hline Fecha: & Área: & \\
\hline \multicolumn{3}{|l|}{ Nombre del Artículo: } \\
\hline Cantidad: & Unidad de medida & Valor S/. \\
\hline \multicolumn{3}{|c|}{ Categoría } \\
\hline 1. Accesorios y herramientas & \multirow{5}{*}{$\begin{array}{l}\text { 6. Cajas de productos } \\
\text { 7. Insumos de mtto } \\
\text { 8. Insumos de embalaje } \\
\text { 9. Equipo de manipulación } \\
\text { 10. Otros (especificar) }\end{array}$} & \\
\hline 2. Instrumentos de medicón & & \\
\hline 3. Residuos de empaques, & & \\
\hline 4. Material y artículo de limpieza & & \\
\hline 5. EPP & & \\
\hline \multicolumn{3}{|c|}{ Razón } \\
\hline 1. Defectuosos & \multirow{3}{*}{$\begin{array}{l}\text { 4. Contaminante } \\
\text { 5. No se necesita pronto } \\
\text { 6. Otros (especificar) }\end{array}$} & \\
\hline 2.No se necesitan & & \\
\hline 3.Material de desperdicio & & \\
\hline \multicolumn{3}{|c|}{ Forma de desecho } \\
\hline 1. Vender & \multirow{2}{*}{$\begin{array}{l}\text { 3. Tirar como basura } \\
\text { 4. Otros }\end{array}$} & \\
\hline 2. Mover de ubicación & & \\
\hline \multicolumn{3}{|l|}{\begin{tabular}{|l|} 
Fecha de desecho: \\
Persona que autoriza \\
\end{tabular}} \\
\hline
\end{tabular}

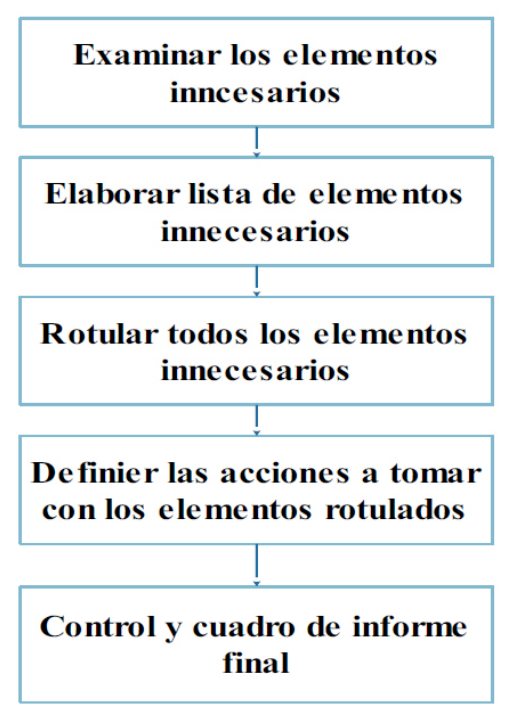

Figura 8. Actividades de etapa de clasificación 


\section{Etapa 2: Implementación Orden-SEITON}

En este paso se procede a ordenar cada artículo en su lugar identificar la ubicación según criterio de frecuencia de uso-eficiencia, peso, seguridad. Ver figura 7.
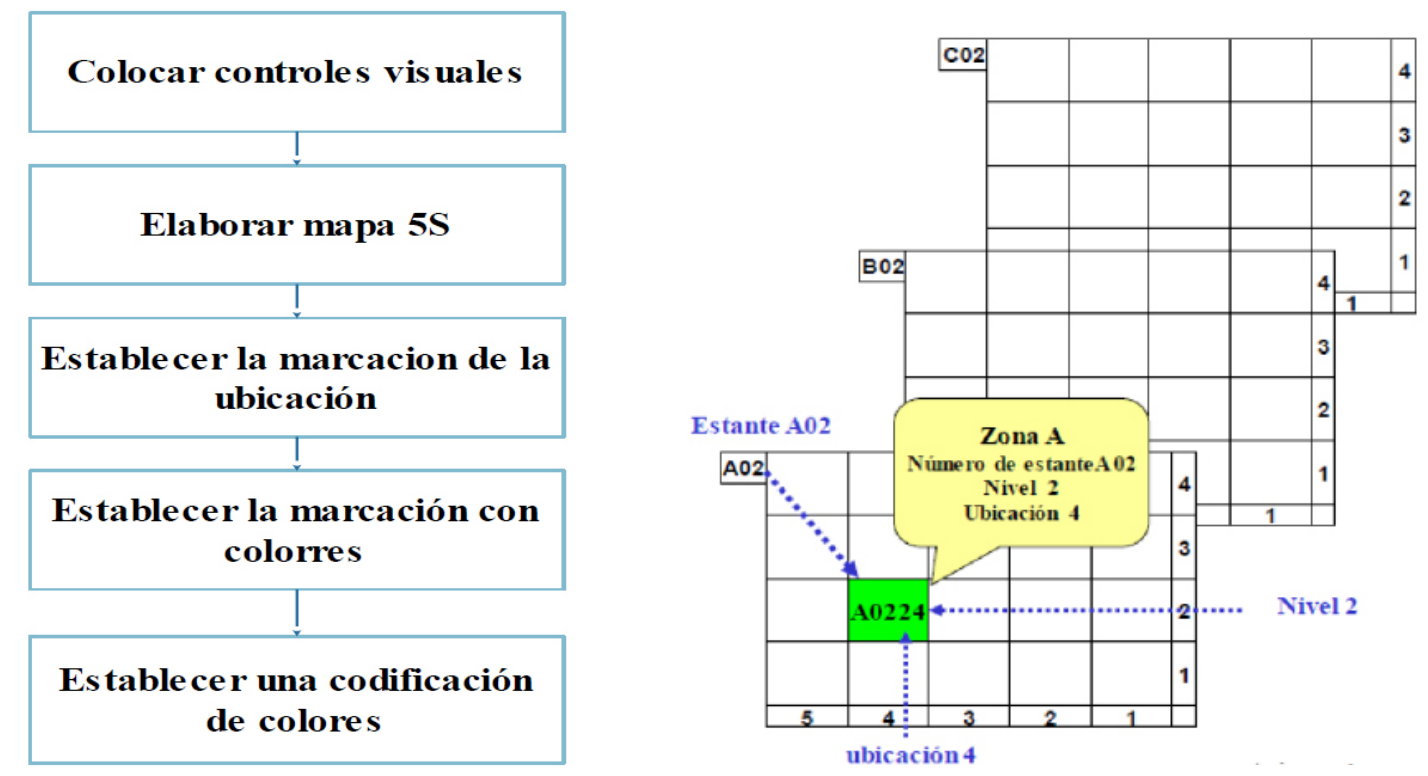

Figura 9. Actividades de etapa de Orden

\section{Etapa 3: implementación de la limpieza - SEISO}

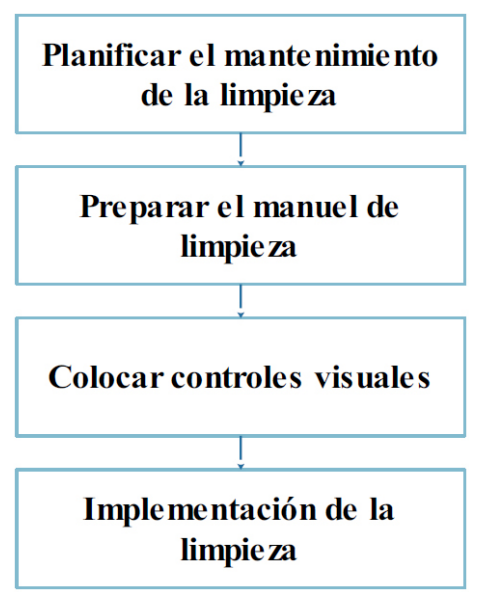

Figura 10. Implementación limpieza

Se procedió a realizar un cronograma de limpieza y responsables de la ejecución asimismo los instrumentos de limpieza para realizar. Por otro lado, se desarrolló un formato de inspección de limpieza para registrar las áreas que tiene mayor incidencia de no realizar la limpieza. Ver Tabla 5. 
Tabla 5. Actividades de etapa de limpieza

\begin{tabular}{|c|c|c|c|}
\hline Uf & \multicolumn{3}{|c|}{ CRONOGRAMA DE LIMPIEZA } \\
\hline Operario & Ubicación & Frecuencia & Materiales necesarios \\
\hline Humberto Bueno & Planta & Supervision diaria & \multirow{7}{*}{$\begin{array}{c}\text { Escoba, recogedor, trapos, } \\
\text { aspiradora etc. }\end{array}$} \\
\hline Juan Ponce & Almacen insumos y final & Diaria & \\
\hline Alejandro Sanchez & Corte y perforado & Diaria & \\
\hline Percy Tarazona & Sellado y empaquetado & Diaria & \\
\hline Milagros carbajal & Compras & Diaria & \\
\hline Marlene Cruz & Ventas & Diaria & \\
\hline Juan Carbs Negron & $\begin{array}{l}\text { Recepcion y patio de } \\
\text { maniobras }\end{array}$ & Diaria & \\
\hline
\end{tabular}

Fuente: Elaboración Propia

Etapa 4: implementación estandarización - SEIKETSU

Con el fin, de garantizar la conservación y se- guir mejorando los resultados obtenidos en las primeras $3 \mathrm{~S}$ se promoverán acciones de estandarización. Ver figura 12.

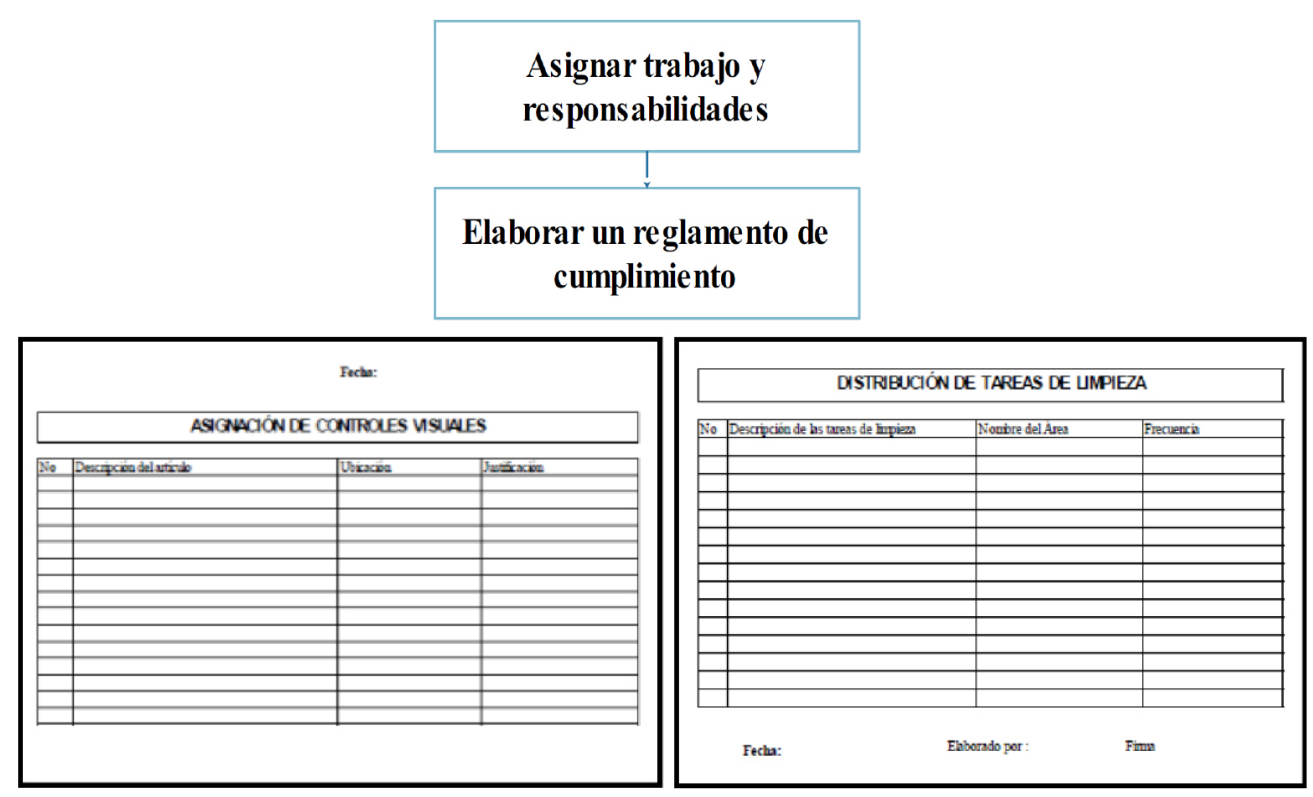

Figura 11. Actividades de etapa de Estandarización Fuente: Elaboración Propia

\section{Etapa 5: Implementación de la Disciplina}

\section{- SHITSUKE}

Es necesario y obligatorio que los gerentes y dueños de la empresa estén comprometidos a la realización de las 55 además fo- mentara que se cumpla los procedimientos y también realizar la auditoria que informara si se está cumpliendo con la implementación se tomara evidencia mediante fotos. Ver figura 9. 


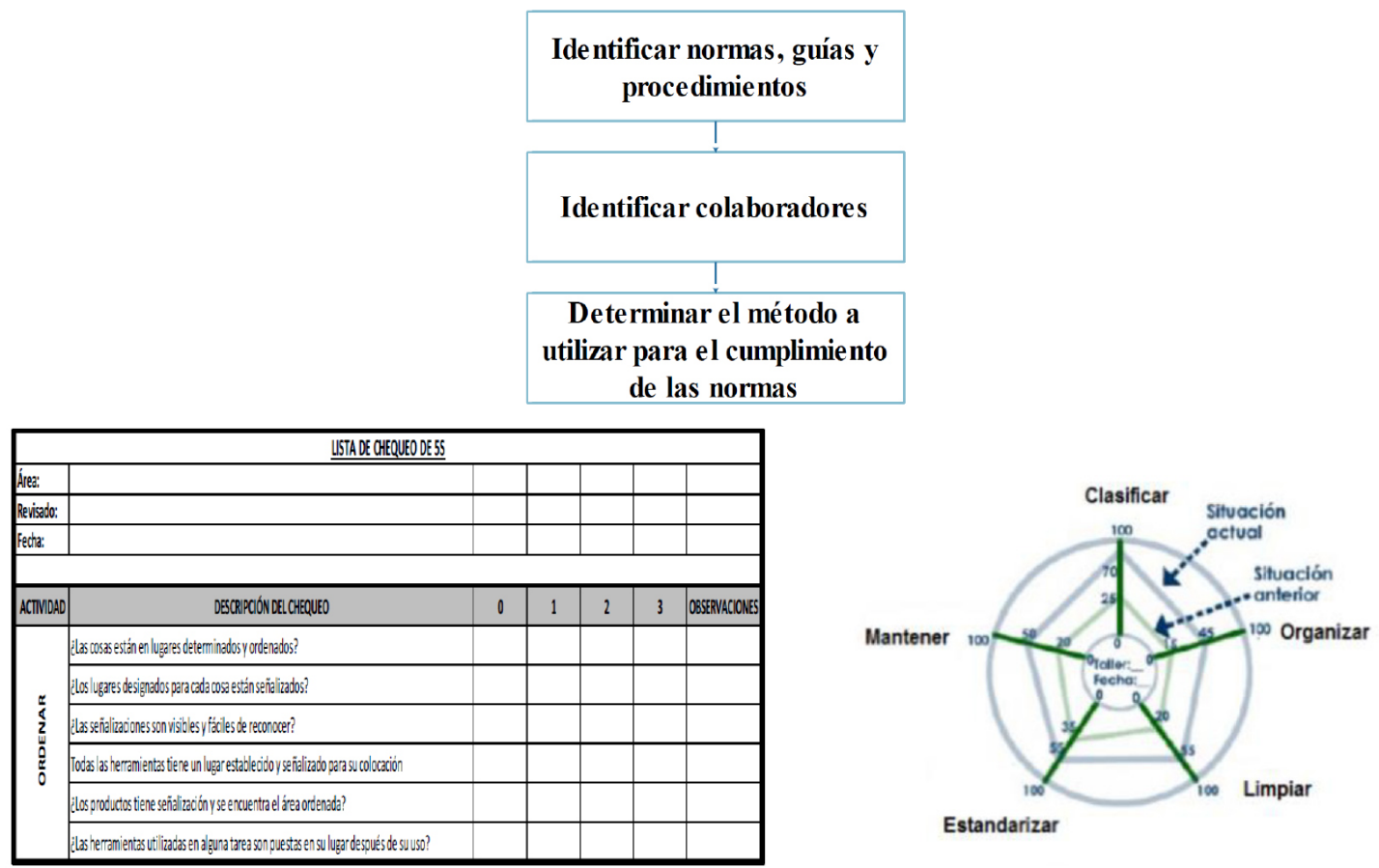

Figura 12. Actividades de etapa de Disciplina

\section{Paso N 7: Control}

Para tener una idea de resumen de los proce- $\quad$ que midan el proceso, en la Tabla 6, se muesdimientos y registros que se implementaron, tra los formatos que permitirán el registro de para la propuesta de mejora los indicadores los indicadores y el control de los procesos.

Tabla 6. Indicadores propuestos para la Imprenta AYEMI E.I.R.L.

\begin{tabular}{cl}
\hline KPI & \multicolumn{1}{c}{ Descripción } \\
\hline & $\%$ de compras rechazados. \\
& $\%$ de proveedores certificados \\
De entrada & $\%$ Órdenes de compras que no cumplan las especificaciones de \\
& calidad \\
& $\%$ de tiempo de entrega de abastecimiento \\
& $\%$ de pérdida por reproceso. \\
De proceso & $\%$ Capacitaciones asistidas del personal. \\
& $\%$ de pedidos rechazados por el cliente. \\
De salida & $\%$ pedidos entregados en el tiempo programado.
\end{tabular}




\section{Análisis Costo - Beneficio}

De acuerdo a las mejoras implementados, se ha calculado la ganancia que se podría obtener para la imprenta AYEMI E.I.R.L. con un total de S/. 97,853.72, tal como se aprecia en la Tabla 7.

Tabla 7. Resumen de los costos antes y después de las mejoras

\begin{tabular}{cccc}
\hline Estado & Total & Reducción & Ganancia \\
\hline Sin Mejora & S/. 136,203.50 & $71.84 \%$ & S/. 97,853.72 \\
Con Mejora & S/. 38,349.78 & & \\
\hline
\end{tabular}

\section{MATERIALES Y MÉTODOS}

En este punto, respecto a la metodología se tiene que el siguiente estudio es de diseño pre experimental, puesto que se implementará la metodología de las 5'S, para evaluar los cambios en la muestra en estudio.

Respecto al tipo de estudio, según lo asumido Tamayo (2012, p.50), indica que existen tres tipos de investigación "descriptivo, histórico y experimental"; siendo para el caso del presente estudio experimental. La población infinita; siendo la muestra de estudio el total de pedidos que fueron entregados fuera de tiempo en el periodo de estudio (año 2018).

\section{RESULTADOS}

En la figura 13, se visualiza el proceso actual sobre la producción de cuadernos universitarios utilizando la simulación Arena.

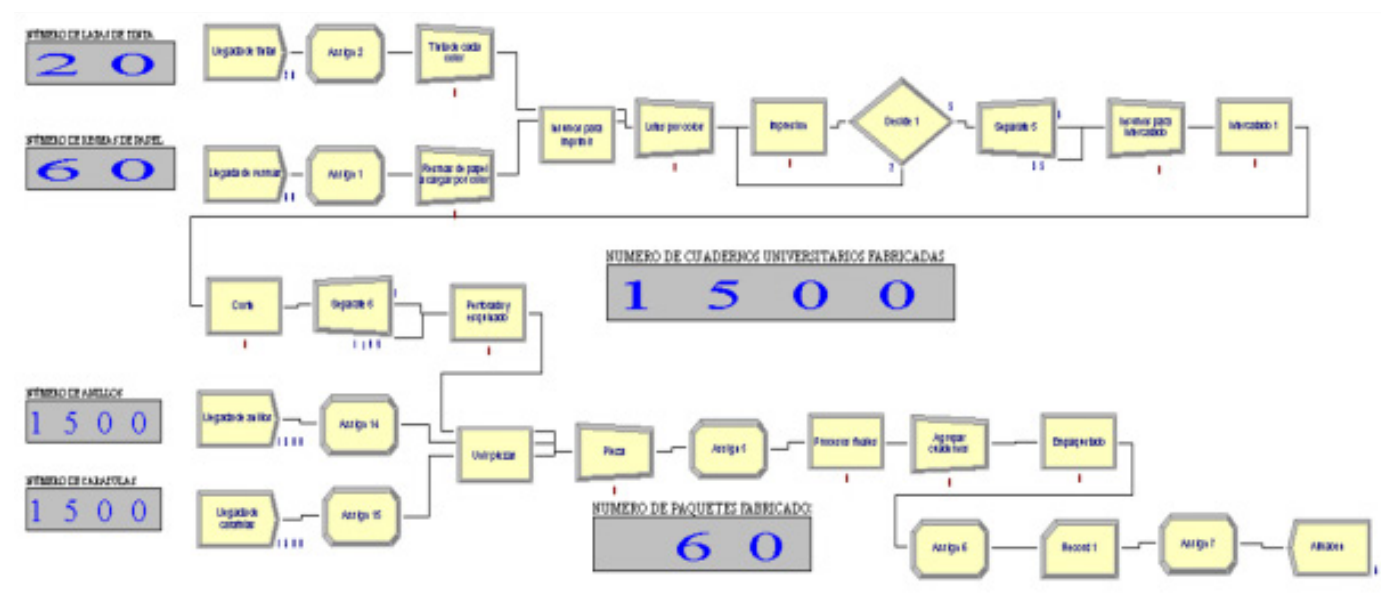

Figura 13. Simulación del proceso actual de la Cadena de Suministro 
En la Figura 13, se realizó la simulación del proceso de la Cadena de Suministro en la pro- ducción de cuadernos universitarios y el total de producción son de 1500 cuadernos.

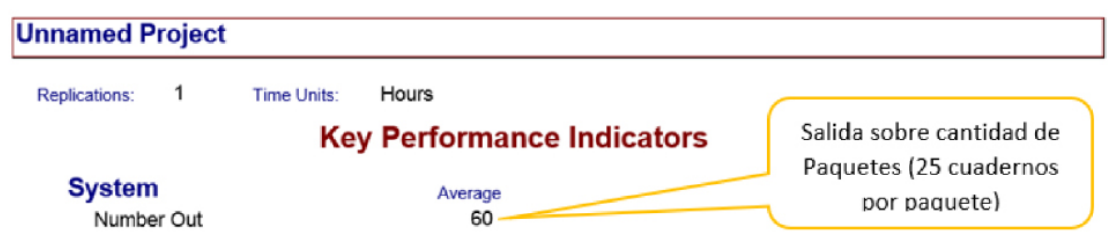

Figura 14. Resultados de la Simulación del Estado Actual

En la Figura 14, se muestra la cantidad de salidas de paquetes en el proceso de producción de cuadernos universitarios. En este caso su puede observar que han salido 60 paquetes que representa 1500 cuadernos.

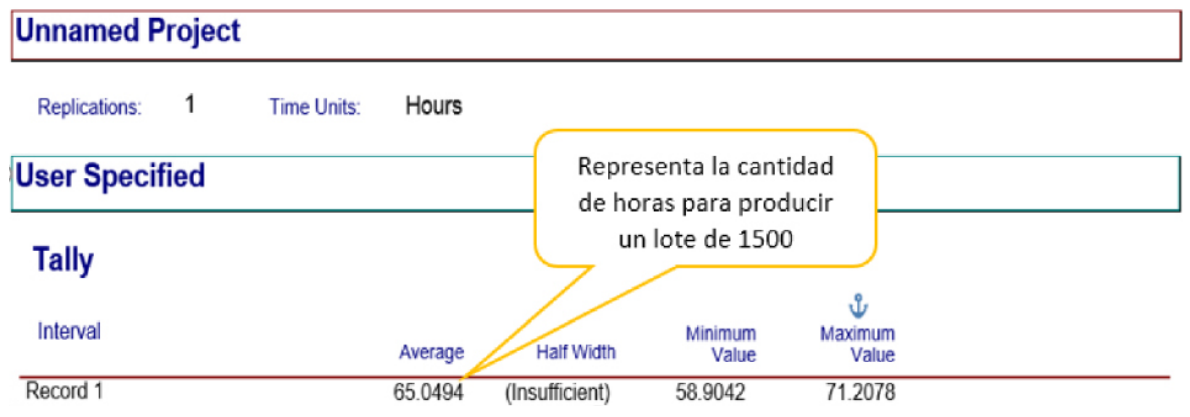

Figura 15. Resultados de la Simulación del Estado Actual

En la Figura 15, se puede apreciar que tiempo que se demoró en producir un lote de 1500 cuadernos universitarios en un total de 65.04 horas que equivale 9 días, las demoras son debidas a las causas que se explicaron en el capítulo 2 que involucra al problema principal que es el incumplimiento con el plazo de entrega de los pedidos de los productos a los clientes.

En la figura 16, se realizó la simulación del proceso de la Cadena de Suministro con la propuesta mejorada de la producción de cuadernos universitarios y el total de producción son de 1500 cuadernos. 


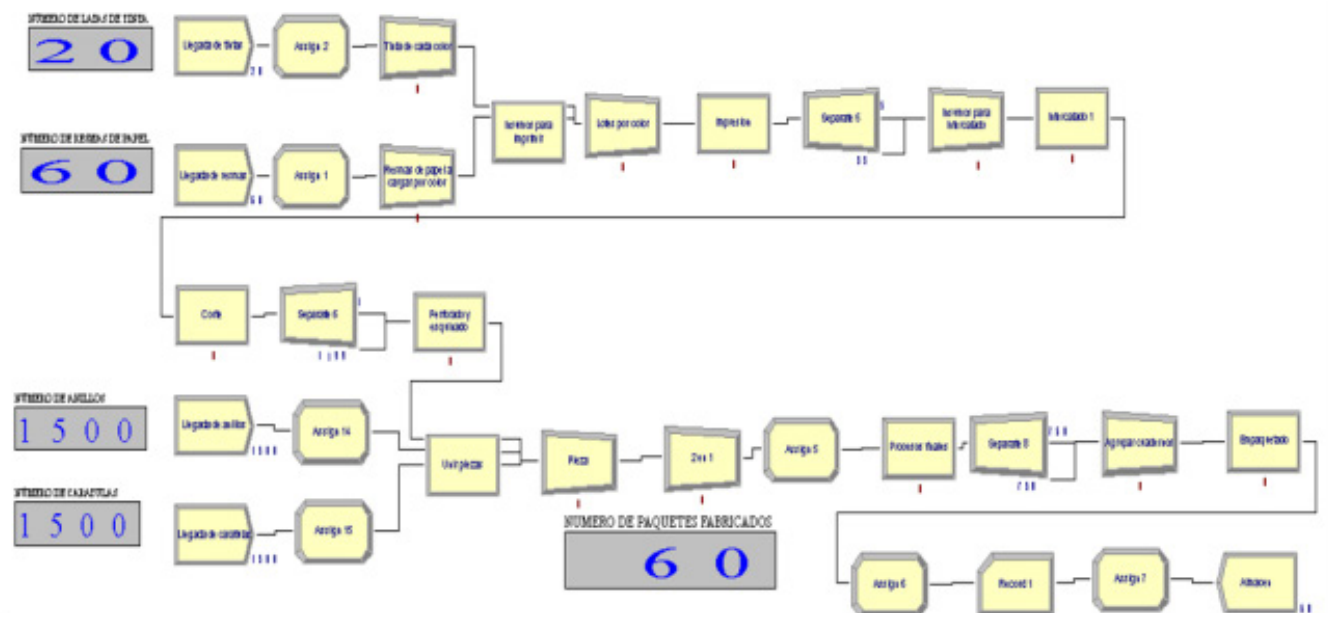

Figura 16. Simulación del proceso propuesto de la Cadena de Suministro

En la Figura 16, se muestra la misma cantidad de salidas de paquetes en el proceso de producción de cuadernos universitarios. En este caso su puede observar que han salido 60 paquetes que representa 1500 cuadernos al igual que el proceso actual.

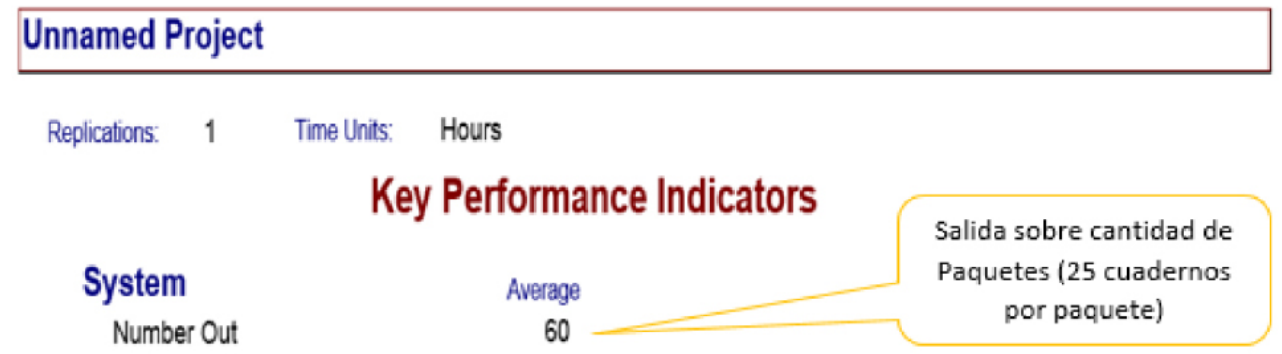

Figura 17. Resultados de la Simulación de la Propuesta Mejorada

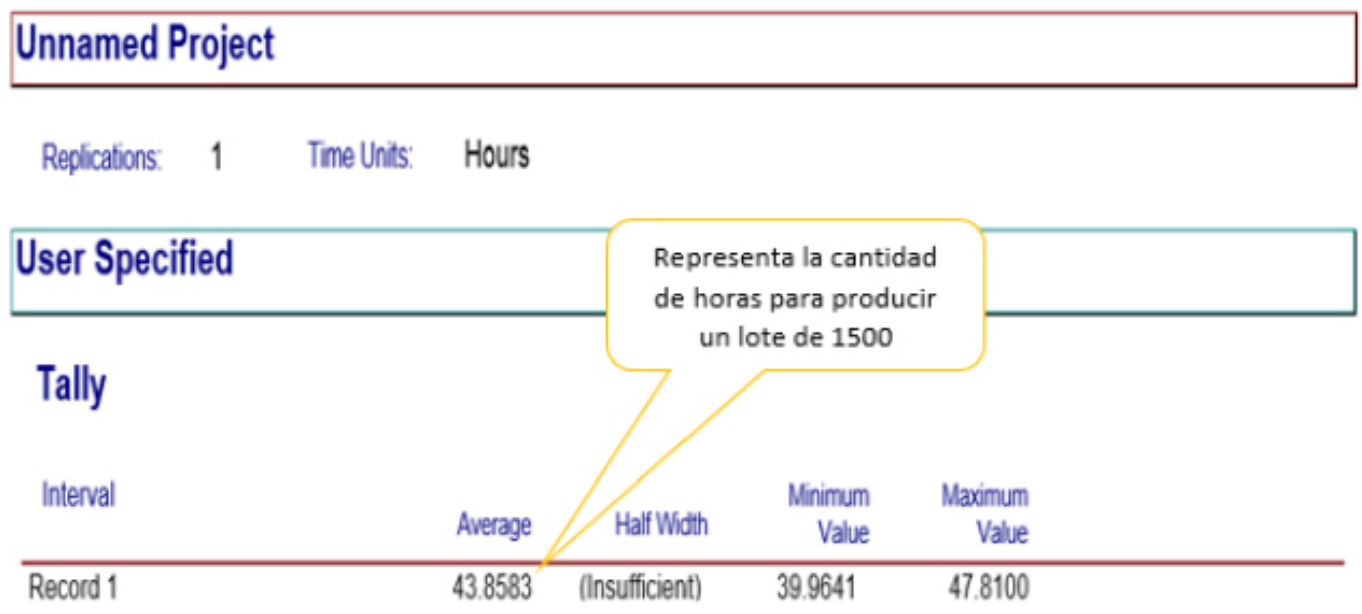

Figura 18. Resultados de la Simulación de la Propuesta Mejorada 
Realizando con la herramienta de la simulación

- El tiempo de proceso de producción actual es de 65.04 horas que equivale 9 días y la propuesta se demora en 43.85 horas que equivale 6 días.

- El tiempo para la ejecución de simulación ha sido establecido como jornada labo ral de 8 horas.

- Con la propuesta hay un ahorro de 3 días.

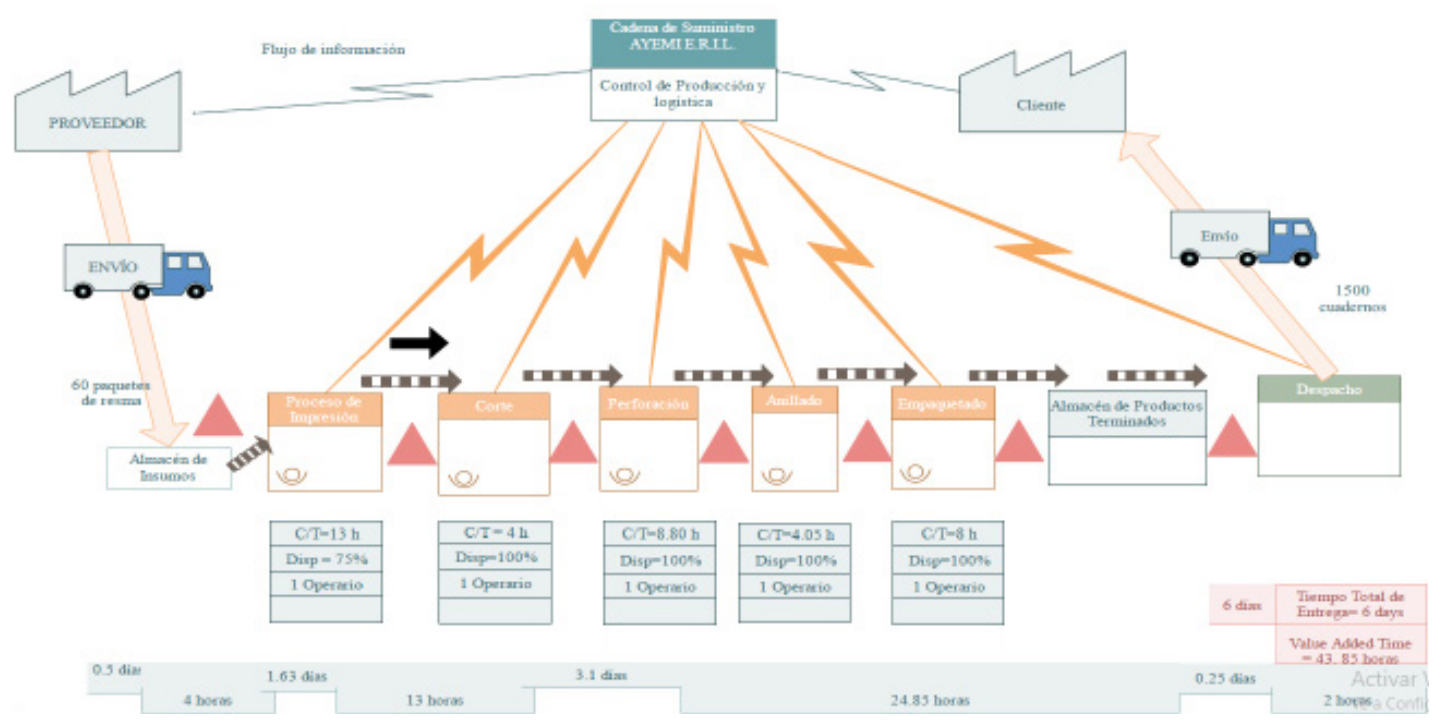

Figura 19. Mapa de Flujo de Valor Mejorado

\section{CONCLUSIONES}

Debido a que se implementó las $5 S$ en el proceso de almacenamiento, esto trajo como impacto positivo la mejora de la cadena de suministro y se logró mejorar la ganancia a un total de S/. 97,853.72 soles.

Con la implementación de las 55 se logró mejorar el ambiente laboral, y el personal mejoró su rendimiento en el almacén de la Imprenta AYEMI E.I.R.L. además de tener ordenado y ubicado correctamente, permite reducir los tiempos en el proceso de producción en un $60 \%$.

La aplicación de la gestión por procesos en la cadena de suministro mejora la gestión del proceso de almacén en la Imprenta AYEMI E.I.R.L., es probado qué la propuesta de mejora logró mejores rendimientos.

Además, se realizó la validación a través del modelo computacional del software de Simulación Arena del proceso actual con respecto al proceso mejorado y teniendo como resultado en un ahorro de 3 días, que representa en la disminución del 33\% y así cumplir con el plazo de entrega de los pedidos.

\section{REFERENCIAS BIBLIOGRÁFICAS}

Camacho, H., Gómez, K., y Monroy, C. (2012). Importancia de la cadena de suministros en las organizaciones. Tenth LACCEI Latin American 
and Caribbean Conference (LACCEI'2012), Megaprojects: Building Infrastructure by fosteringengineering collaboration, efficient and effective integration and innovative planning, July 23-27, 2012, Panama City, Panama.

Cano, P., Orue, F., Martínez, J., Mayett J., y López, G. (2015). Modelo de gestión logística para pequeñas y medianas empresas en México. Contaduría y Administración 60 (1), enero-marzo 2015: 181-203.

Ramírez, S., y Peña., G. (2011). Análisis de comportamiento caótico en variables de la cadena de suministro. Journal of Economics, Finance and Administrative Science v.16 n.31 Lima

Salas, K., Maiguel, H., y Acevedo, J. (2017). Metodología de Gestión de Inventarios para determinar los niveles de integración y colaboración en una cadena de suministro. Ingeniare. Revista Chilena de Ingeniería, vol. 25, núm. 2, junio, 2017, pp. 326-337. Universidad de Tarapacá. Arica, Chile.

Salas, K., Meza, J., Obredor, T., y Mercado, N. (2019). Evaluación de la Cadena de Suministro para Mejorar la Competitividad y Productividad en el Sector Metalmecánico en Barranquilla, Colombia. Inf. tecnol. vol.30 no.2 La Serena.

Tamayo, T. (2012). El proceso de Investigación Científica. México: Limusa Noriega Editores. 5a. Ed.

Vianchá, Z. (2014). Modelos y configuraciones de cadenas de suministro en productos perecederos. Ingeniería y Desarrollo, vol. 32, núm. 1, enero-junio, 2014, pp. 138-154. Universidad del Norte.

\section{CORRESPONDENCIA:}

Dr. Jorge Rafael Diaz Dumont jorgediaz@unat.edu.pe 